Pacific Journal of Mathematic 


\title{
LATTICE VARIETIES COVERING THE SMALLEST NON-MODULAR VARIETY
}

\author{
BJARNI Jónsson AND IVAN RIVAL
}

\begin{abstract}
There are sixteen varieties of lattices that are known to cover $N$, the variety generated by the five-element nonmodular lattice $N$. Fifteen of these are generated by finite subdirectly irreducible lattices $L_{1}, L_{2}, \cdots, L_{15}$, and the sixteenth is jointly generated by $N$ and the diamond $M_{3}$. We show that every variety of lattices that properly contains $N$ includes one of the lattices $M_{3}, L_{1}, L_{2}, \cdots, L_{15}$. Of these sixteen lattices, the first six fail to be semidistributive; in fact, every variety of lattices in which the semidistributive law fails contains one of these six.
\end{abstract}

1. Introduction. By a variety of lattices is meant the class of all those lattices satisfying some fixed set of lattice identities. With respect to set inclusion the set of all varieties of lattices itself constitutes a lattice. The least element of this lattice is the class of all one-element lattices and the greatest element is the class of all lattices. Moreover, this lattice is distributive [5] and it has cardinality $2^{\aleph_{0}}[1],[7]$.

Let $K$ denote a class of lattices and let $\boldsymbol{K}$ denote the variety generated by $K$. To determine $K$ by finding all of the identities that hold in every lattice in $K$ is often very difficult. On the other hand, there is an alternative approach to the problem of describing $\boldsymbol{K}$ which stems from the well known fact, due to G. Birkhoff, that a variety of lattices is determined by its subdirectly irreducible members. In fact, it is customary, where possible, to identify a given variety of lattices with its subdirectly irreducible members. For instance, in the lattice of varieties of lattices there is a unique atom whose only subdirectly irreducible member is the two-element chain: the variety of all distributive lattices. Covering this variety are precisely two varieties: one is $\boldsymbol{M}_{3}$, the variety generated by the diamond, $M_{3}$ (the five element modular non-distributive lattice); the other is $N$, the variety generated by the pentagon $N$ (the fiveelement non-modular lattice). While there is a great deal known about varieties of modular lattices (for instance, that the least modular variety $\boldsymbol{M}_{3}$ is covered by precisely three varieties, each generated by its finite subdirectly irreducible members [6] (cf. [4])) the non-modular case has proved to be more difficult to describe.

In [8] R. McKenzie lists fifteen finite, subdirectly irreducible, non-modular lattices $L_{1}, L_{2}, \cdots, L_{15}$ (Fig. 1) each of which generates 


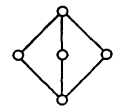

$M_{3}$
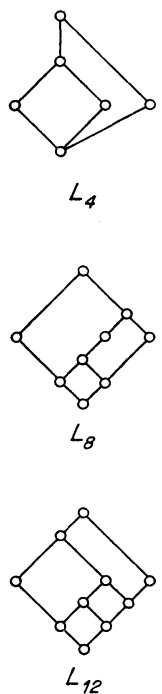

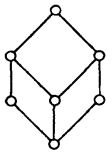

$L_{1}$

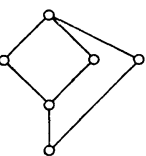

$L_{5}$
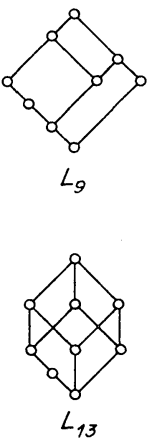

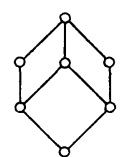

$L_{2}$

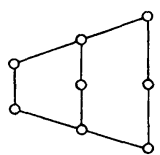

$L_{6}$
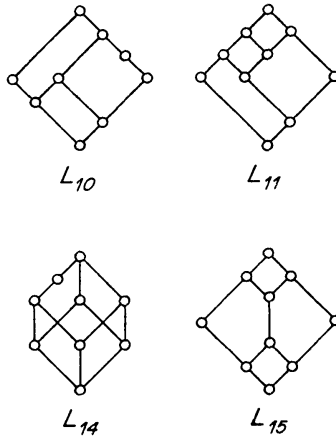

Figure 1

a variety that covers $N$. A sixteenth cover is jointly generated by $N$ and $M_{3}$. Our principal result shows that McKenzie's list is complete.

THEOREM 1.1. Every variety of lattices that properly contains $N$ includes one of the lattices $M_{3}, L_{1}, L_{2}, \cdots, L_{15}$.

This theorem was first established by I. Rival [9] under the additional assumption that the variety in question is generated by a lattice in which every chain is finite. Subsequently, B. Jonsson succeeded in removing this condition.

The proof of Theorem 1.1 consists of three main parts corresponding to a cumulative classification of the lattices $M_{3}, L_{1}, L_{2}, \cdots, L_{15}$.

The first part concerns semidistributivity. A lattice $L$ is semidistributive if, for all $u, v, x, y, z \in L, u=x+y=x+z$ implies $u=x+y z$, and dually, $v=x y=x z$ implies $v=x(y+z)$. Call a variety of lattices semidistributive if each of its members is semidistributive. The main result of this part of the proof is of some independent interest.

THEOREM 1.2. A variety of lattices is semidistributive if and 
only if it contains none of the lattices $M_{3}, L_{1}, L_{2}, L_{3}, L_{4}$, and $L_{5}$.

This result was first proved by B.A. Davey, W. Poguntke and I. Rival [2] for those varieties generated by a lattice satisfying the double chain condition.

The second part of the proof concerns the behavior of congruence relations in non-modular lattices. Let $a, b$, and $c$ be elements of a lattice $L$ which generate a pentagon; that is, $b c<a<c<a+b$. We write $N(a, b, c)$ to indicate that this relation holds. Call a quotient $c / a$ of $L$ an $N$-quotient if $N(a, b, c)$ for some $b$. Let $L$ be a lattice in a semidistributive variety that contains none of the lattices $L_{6}, L_{7}, L_{8}, L_{9}, L_{10}, L_{11}$, and $L_{12}$. The basic theme of this part of the proof is that projectivities between $N$-quotients in $L$ behave like projectivities between quotients in a distributive lattice.

The final part concerns critical edges of a subdirectly irreducible lattice $L$. We call a quotient $c / a$ of a subdirectly irreducible lattice $L$ a critical edge if every non-trivial congruence relation on $L$ identifies $a$ and $c$. Let $V$ be a variety that contains none of the lattices $M_{3}, L_{1}, L_{2}, \cdots, L_{12}$ and let $L \in V$ be subdirectly irreducible and nondistributive. We prove that $L$ has a unique critical edge $c / a$, that $c / a$ is the only $N$-quotient of $L$, and that the smallest congruence relation $\operatorname{con}(a, c)$ which identifies $a$ and $c$ identifies no two distinct elements besides $a$ and $c$. Moreover, $L / \operatorname{con}(a, c)$ is distributive (cf. $L=L_{13}, L_{14}$ or $L_{15}$ ).

Therefore $L$ is locally finite, and since every variety is determined by its finitely generated subdirectly irreducible members, we may assume that $L$ is finite. It is now only a matter of straightforward calculations to show that if $V$ does not contain $L_{13}, L_{14}$ or $L_{15}$ then $L$ must be a pentagon.

The final section of this paper is devoted to several results related to Theorem 1.1.

We are indebted to $\mathrm{Mr}$. Wilfried Ruckelshausen, who called our attention to a gap in one of our proofs, and also pointed out simplifications of two other arguments.

2. Semidistributivity. The principal aim of this section is the proof of Theorem 1.2. This generalization of the main result of [2] is realized by focussing attention on the lattices $L^{\sigma}$, of all ideals of $L$, and $L^{\pi}$, of all dual ideals of $L$. Of course, each of $L, L^{\sigma}$, and $L^{\pi}$ generates the same variety of lattices. Moreover, $L$ is embeddable in both $L^{\sigma}$ and $L^{\pi}$. The advantage of $L^{\sigma}$ over $L$ lies in the fact that $L^{\sigma}$ is compactly generated, whence weakly atomic. For instance, for $a, b \in L$ there exists an element $c$ in $L^{o}$ such that $a \leqq c$ and which is covered by $a+b(c<a+b)$. 
Theorem 1.2 is an immediate consequence of the following result.

LEMMA 2.1. If the lattice $L$ is not semidistributive, then either $L^{\sigma \pi \sigma}$ or $L^{\pi \sigma \pi}$ contains a sublattice isomorphic to one of the lattices $M_{3}, L_{1}, L_{2}, L_{3}, L_{4}$ or $L_{5}$.

Proof. Let us suppose that the semidistributive law fails in $L$. By duality we may assume that there exist $u, x, y, z \in L$ such that

$$
u=x+y=x+z,
$$

but not $u=x+y z$. We claim that in the larger lattice $L^{\sigma z}$ we can find elements $u, x, y, z$ that satisfy not only (1), but also

$$
y z \leqq x \prec u, x y \prec y, x z \prec z .
$$

In fact, given elements $u, x, y, z \in L$ such that (1) holds and $x+y z<u$, we can find $x^{\prime} \in L^{\sigma}$ such that $x+y z \leqq x^{\prime} \prec u$, and we therefore have

$$
u=x^{\prime}+y=x^{\prime}+z, y z \leqq x^{\prime} \prec u .
$$

In $L^{\sigma \pi}$ we can then find minimal elements $y^{\prime}$ and $z^{\prime}$ subject to the conditions $u=x^{\prime}+y^{\prime}=x^{\prime}+z^{\prime}, y^{\prime} \leqq y, z^{\prime} \leqq z$. Then $x^{\prime} y^{\prime}<y^{\prime}$. Furthermore, if $x^{\prime} y^{\prime}<t \leqq y^{\prime}$, then $x^{\prime}<x^{\prime}+t \leqq u$ and hence $u=x^{\prime}+t$, so that $t=y^{\prime}$. Thus, $y^{\prime}$ covers $x^{\prime} y^{\prime}$ and, similarly, $z^{\prime}$ covers $x^{\prime} z^{\prime}$. Therefore (1) and (2) are satisfied if we replace $x, y$, and $z$ by $x^{\prime}, y^{\prime}$, and $z^{\prime}$.

We now assume that the elements $u, x, y, z \in L^{\sigma \pi}$ satisfy (1) and (2), and begin by looking at the sublattice generated by $y, z, x y$, and $x z$. In view of (2) we have

$$
\begin{array}{lll}
y \leqq x y+z & \text { or } & y(x y+z)=x y \\
z \leqq x z+y & \text { or } & z(x z+y)=x z
\end{array}
$$

Of the four cases that arise, three easily yield one of the lattices $L_{i}, i \leqq 5$, as a sublattice of $L^{\sigma \pi}$. First, let $y \leqq x y+z$ and $z \leqq x z+y$. Let $v=x y+x z$, and observe that $y \not \leq x$ and $z \not \leq x$, hence $y \not \leq v$ and $z \nsubseteq v$. Consequently, $y v=x y$ and $z v=x z$. Also, $y+z=$ $y+v=z+v$, and, therefore, $L_{2}$ is a sublattice of $L^{\sigma \pi}$ (Fig. 2).

Next, let us suppose that $y(x y+z)=x y$ and $z \leqq x z+y$. The lattice generated by $y, z, x y$, and $x z$ is a homomorphic image of the lattice in (Fig. 3). Let $v=x y+z$. If $x(y+z)+v=y+z$, then $y, v$, and $x(y+z)$ generate a lattice isomorphic to $L_{5}$, or to $M_{3}$ if $x v=x y$, while if $x(y+z)+v<y+z$, then $x, y$, and $x(y+z)+v$ generate a lattice isomorphic to $L_{3}$ (Fig. 4). The case in which $y \leqq x y+z$ and $z(x z+y)=x z$ is symmetric to the preceding case, 
and it remains, therefore, to consider only the case in which $y(x y+z)=x y$ and $z(x z+y)=x z$.

Let $y_{0}=y$ and $z_{0}=z$, and, for $n=0,1, \cdots$, let

$$
y_{n+1}=y+x z_{n}, z_{n+1}=z+x y_{n} .
$$

Then (1) obviously holds with $y$ and $z$ replaced by $y_{n}$ and $z_{n}$. Denote by $\left(2_{n}\right)$ the formula obtained from (2) be replacing $y$ and $z$ by $y_{n}$ and $z_{n}$. Suppose $\left(2_{n}\right)$ holds, and consider $\left(2_{n+1}\right)$. We may assume that $y_{n} z_{n+1}=x y_{n}$ and $z_{n} y_{n+1}=x z_{n}$, for otherwise one of the three cases already considered would apply with $y$ and $z$ replaced by $y_{n}$ and $z_{n}$. As before, we can assume that $y_{n}\left(x y_{n}+z_{n}\right)=x y_{n}$ and $z_{n}\left(x z_{n}+y_{n}\right)=x z_{n}$, for otherwise we are done. We have $z \leqq z_{n+1}$ and $z \not x$, so $z_{n+1} \not \leq x$, and hence, $x z_{n+1}<z_{n+1}$. If $x z_{n+1}<t<z_{n+1}$, then the elements, $x, z_{n}$ and $t$ generate a lattice isomorphic to $L_{3}$ (Fig. 5). We may, therefore, assume that $x z_{n+1} \prec z_{n+1}$ and, similarly, $x y_{n+1} \prec y_{n+1}$. We may also assume that $y_{n+1} z_{n+1} \leqq x$, for otherwise $y_{n}, x y_{n+1}$ and $y_{n+1} z_{n+1}$ generate a lattice isomorphic to $L_{5}$. Thus, we may assume that $\left(2_{n}\right)$ holds for all $n$.

In $L^{\sigma \pi \sigma}$ we now form the join $y_{\infty}$ of all the elements $y_{n}$, and the join $z_{\infty}$ of all the elements $z_{n}$. Obviously

$$
u=x+y_{\infty}=x+z_{\infty}, y_{\infty}+z_{\infty}=y+z .
$$

Furthermore, $x \notin y_{n}$ for all $n$ and, therefore, $x \notin y_{\infty}$. Thus, $x y_{\infty}<y_{\infty}$, and since $x y_{n} \prec y_{n}$ for all $n$ we have in fact that $x y_{\infty} \prec y_{\infty}$; similarly $x z_{\infty} \prec z_{\infty}$. Finally, from the fact that $x y_{n}+x z_{n} \leqq y_{n+1} z_{n+1} \leqq x$ for all $n$ we infer that $x y_{\infty}=x z_{\infty}=y_{\infty} z_{\infty}$.

Dropping the subscripts in order to simplify the notation, we now have four elements $u, x, y$, and $z$ in $L^{\sigma \pi \sigma}$ that satisfy (1) and (2) and, in addition, $x y=x z=y z$. Letting $v=x(y+z)$, we consider four cases depending on whether or not the equations $y+z=y+v$ and $y+z=z+v$ hold. If both equations fail, then the elements $y, z, y+v$, and $z+v$ generate a homomorphic image of $L_{1}$ (Fig. 6). We may assume that this is a proper homomorphism, so that $v=y z$; then $x, y$, and $z$ generate a lattice isomorphic to $L_{4}$. If just one equation holds, say, $y+z=y+v>z+v$, then $y$, $z$, and $v$ generate a lattice isomorphic to $L_{4}$. Finally, if both equations hold, then $y, z$, and $v$ generate a diamond.

This completes the proof of Lemma 2.1, and therefore also the proof of Theorem 1.2.

The remainder of this section is concerned with the behavior of congruence relations in a semidistributive lattice. We first dispense with the necessary preliminaries.

Given two quotients $p / q$ and $r / s$ in a lattice $L$ if $r=p+s$ and 
$s \geqq q$ then we say that $p / q$ transposes weakly $u p$ onto $r / s$ and that $r / s$ is a weak upper transpose of $p / q$, -in symbols $p / q \nearrow_{w} r / s,-$ and we refer to the map $t \rightarrow t+s(t \in p / q)$ as a weak upper transposition. Dually, if $q r=s$ and $r \leqq p$ then we say that $p / q$ transposes weakly down onto $r / s$ and that $r / s$ is a weak lower transpose of $p / q$, -in symbols $p / q \searrow_{w} r / s$, - and we refer to the map $t \rightarrow t r(t \in p / s)$ as a weak lower transposition of $p / q$ onto $r / s$. If there exists a sequence of quotients $x_{0} / y_{0}, x_{1} / y_{1}, \cdots, x_{n} / y_{n}$ with $x_{0} / y_{0}=p / q$ and $x_{n} / y_{n}=r / s$ such that, for each $i<n, x_{i} / y_{i}$ transposes weakly up or down onto $x_{i+1} /$ $y_{i+1}$, then we say that $p / q$ projects weakly onto $r / s$, and we refer to the composition of the weak transpositions of $x_{i} / y_{i}$ onto $x_{i+1} / y_{i+1}$ for $i=0,1, \cdots, n-1$ as a weak projectivity of $p / q$ onto $r / s$.

If both $p / q \nearrow_{w} r / s$ and $r / s \searrow_{w} p / q$, that is, if $p+s=r$ and $p s=q$, then we say that $p / q$ transposes $u p$ onto $r / s$ and that $r / s$ transposes down onto $p / q$, - in symbols $p / q \nearrow r / s$ and $r / s \searrow p / q$, -and we say that $r / s$ is an upper transpose of $p / q$ and $p / q$ is a lower transpose of $r / s$. In this case the maps $t \rightarrow t+s(t \in p / q)$ and $t \rightarrow t p(t \in r / s)$ are referred to as an upper transposition of $p / q$ onto $r / s$ and a lower transposition of $r / s$ onto $p / q$, respectively. If there exists a sequence of quotients $x_{0} / y_{0}, x_{1} / y_{1}, \cdots, x_{n} / y_{n}$ with $x_{0} / y_{0}=p / q$ and $x_{n} / y_{n}=r / s$ such that, for each $i<n, x_{i} / y_{i}$ transposes up or down onto $x_{i+1} / y_{i+1}$, then $p / q$ is said to project onto $r / s$, and the composition of the transportations of $x_{i} / y_{i}$ onto $x_{i+1} / y_{i+1}$ for $i<n$ is called a projectivity of $p / q$ onto $r / s$.

Our next lemma concerns the possibility of shortening a sequence of weak transpositions. Let us suppose that $p / q$ projects weakly onto $r / s$ in $n$ steps, say

$$
p / q=x_{0} / y_{0} \nearrow_{w} x_{1} / y_{1} \succ_{w} x_{2} / y_{2} \nearrow_{w} \cdots x_{n} / y_{n}=r / s .
$$

Let $n>2$. If there exists a quotient $u / v$ such that

$$
x_{0} / y_{0} \succ_{w} u / v \succ_{w} x_{2} / y_{2},
$$

then we can shorten the sequence of weak transpositions by replacing the two quotients $x_{1} / y_{1}$ and $x_{2} / y_{2}$ by the single quotient $u / v$. In a distributive lattice this can always be done, and the non-existence of such a quotient $u / v$ is therefore connected with the presence of a diamond or a pentagon as a sublattice of $L$. If $L$ is semidistributive, then this sublattice must of course be a pentagon. The aim of the lemma is to describe the location of the pentagon relative to the quotients $x_{i} / y_{i}$.

LeMma 2.2. Let $L$ be a semidistributive lattice, and let $x_{0} / y_{0}$, $x_{1} / y_{1}$, and $x_{2} / y_{2}$ be quotients in $L$ such that $x_{0} / y_{0} /{ }_{w} x_{1} / y_{1} \searrow_{w} x_{2} / y_{2}$. Then either there exists a subquotient $p / q$ of $x_{0} / y_{0}$ such that, for 


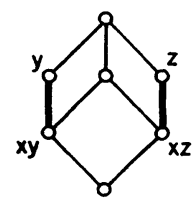

Fig. 2

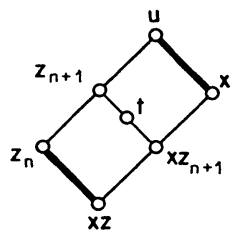

Fig. 5

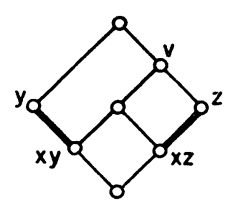

Fig. 3

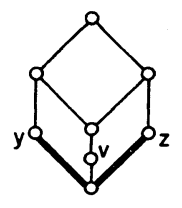

Fig. 6

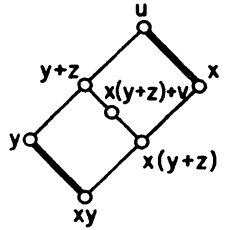

Fig. 4

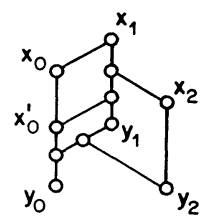

Fig. 7

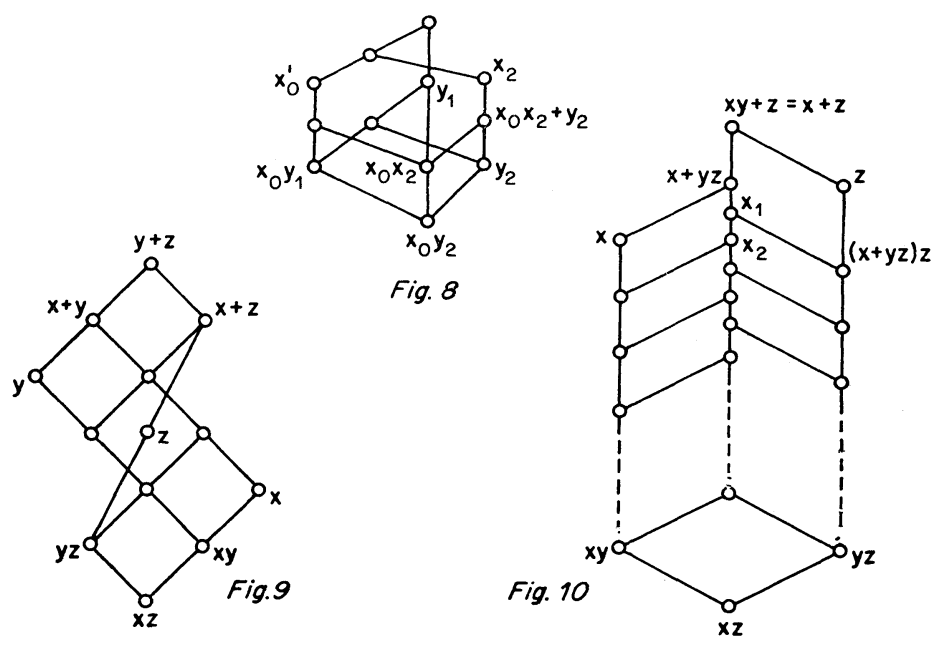

some quotient $u / v, p / q \backslash u / v \nearrow x_{2} / y_{2}$, or else there exist $a, b, c \in L$ with $N(a, b, c)$ such that either $b / b c$ is a subquotient of $x_{0} / y_{0}$, or else $(a+b) / b$ transposes down onto a subquotient of $x_{0} / y_{0}$.

Proof. Let $x_{0}^{\prime}=x_{0}\left(y_{1}+x_{2}\right)$. If $x_{0}^{\prime}+y_{1}<x_{2}+y_{1}$, then the elements $a=x_{0}^{\prime}+y_{1}, b=x_{0}$ and $c=y_{1}+x_{2}$ satisfy $N(a, b, c)$, and $b / b c=$ $x_{0} / x_{0}^{\prime}$ is a subquotient of $x_{0} / y_{0}$ (Fig. 7).

Let $x_{0}^{\prime}+y_{1}=x_{2}+y_{1}$. By the semidistributivity of $L, x_{2}+y_{1}=$ $x_{0}^{\prime} x_{2}+y_{1}=x_{0} x_{2}+y_{1}$. If $x_{0} x_{2}+y_{2}<x_{2}$, then the elements $a=x_{0} x_{2}+y_{2}$, $b=y_{1}$ and $c=x_{2}$ satisfy $N(a, b, c)$, and $(a+b) / b$ transposes down onto the subquotient $x_{0}^{\prime} / x_{0} y_{1}$ of $x_{0} / y_{0}$ (Fig. 8).

Finally, if $x_{0} x_{2}+y_{2}=x_{2}$, then the subquotient $\left(x_{0} y_{1}+x_{0} x_{2}\right) / x_{0} y_{1}$ of $x_{0} y_{0}$ transposes down onto the quotient $x_{0} x_{2} / x_{0} y_{2}$, which transposes up onto $x_{2} / y_{2}$. 
3. Projectivities between $N$-quotients. Consider a variety $\boldsymbol{V}$ that contains none of the lattices $M_{3}, L_{1}, L_{2}, \cdots, L_{12}$ and a lattice $L \in V$. Our aim in this section is to show that projectivities between $N$-quotients in $L$ behave like projectivities between quotients in a distributive lattice.

To this end we require a preliminary result concerning lattices determined by defining relations (relative to the variety of all lattices). The result is most easily formulated by means of a diagram; indeed, the proof itself becomes quite transparent when presented pictorially.

LEMMA 3.1. Let $L$ be a semidistributive lattice generated by three elements $x, y$, and $z$, with $x \leqq x y+z$ and $x z \leqq y$. If $L$ does not have a sublattice isomorphic to $L_{7}, L_{8}$ or $L_{12}$, then $L$ is a homomorphic image of the lattice in Fig. 9.

Proof. It is easy to check that Fig. 9 represents the lattice with the defining relations $x \leqq x y+z, x z \leqq y,(x+y) z=y z,(x+y z) y=$ $x y+y z$, and $x+y(x+z)=(x+y)(x+z)$. It therefore suffices to show that under the hypotheses of the lemma the last three of these relations hold.

The lattice determined by $x, z, x y$, and $y z$ and the defining relations $x \leqq x y+z$ and $x z \leqq y$ (relative to the variety of all lattices) is pictured in Fig. 10. In order to avoid $L_{12}$ we must have $x_{1}=x_{2}$, where $x_{1}=x y+(x+y z) z$ and $x_{2}=y z+x x_{1}$. Since $(x y+y z)+x_{2} x=$ $x_{2}=x_{1}=(x y+y z)+(x+y z) z$, semidistributivity yields $x_{2}=(x y+y z)+$ $x_{2} x(x+y z) z=x y+y z$. As $z(x y+y z)=y z$, we conclude that $(x+y z) z=(x+y z) z x_{1}=(x+y z) z(x y+y z)=y z$. Hence, by the semidistributivity of $L,(x+y) z=y z$. Next, check that the elements $x, z, x y, y z$ and $(x+y z) y$ generate a homomorphic image of the lattice in Fig. 11. To avoid $L_{8}$ we must therefore have $(x+y z) y=x y+y z$. Finally, observe that the elements $y, z, x+y$, $x+z$ and $x+y(x+z)$ generate a homomorphic image of the lattice in Fig. 12. To avoid $L_{7}$ we must therefore have $x+y(x+z)=$ $(x+y)(x+z)$.

For the remainer of this section let $L$ be a lattice in a variety that contains none of the lattices $M_{3}, L_{1}, L_{2}, \cdots, L_{12}$.

LemMA 3.2. If $a, b, c, a^{\prime}, c^{\prime} \in L, N(a, b, c)$, and $c / a / c^{\prime} / a^{\prime}$, then $N\left(a^{\prime}, b, c^{\prime}\right)$ and, for all $t \in c / a$ and $t^{\prime} \in c^{\prime} / a^{\prime},\left(t+a^{\prime}\right) c=t$ and $t^{\prime} c+a^{\prime}=t^{\prime}$.

Proof. We have $c a^{\prime}=a$ and $c+a^{\prime}=c^{\prime}$. Taking $x=c, y=a^{\prime}$, 


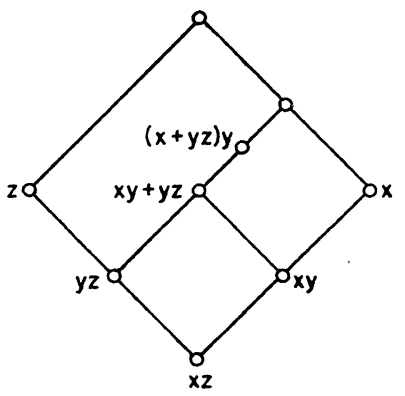

Fig. 11

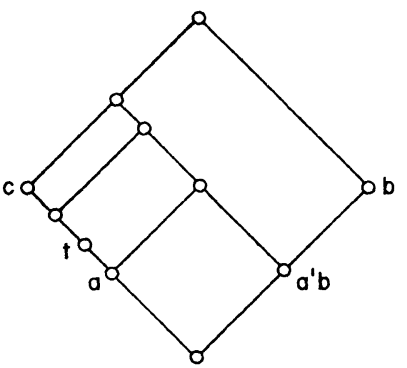

Fig. 13

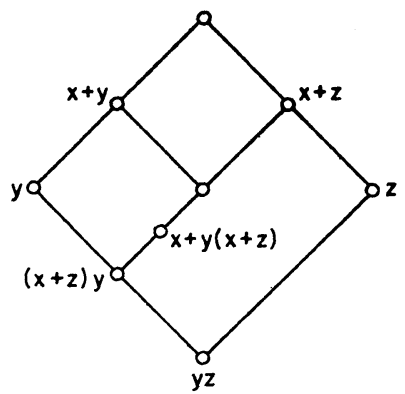

Fig. 12

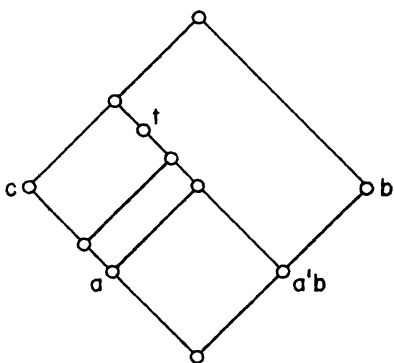

Fig. 14

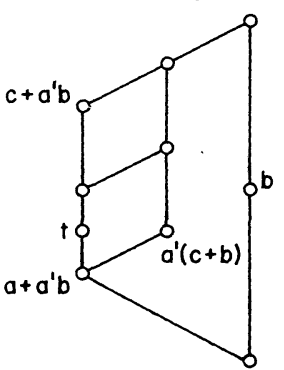

Fig. 15

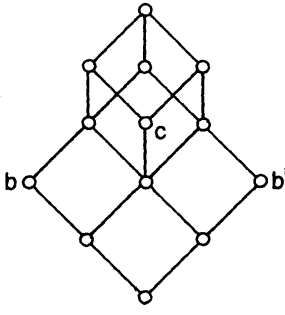

Fig. 16

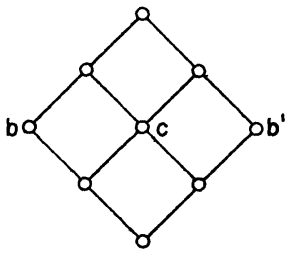

Fig. 17

and $z=b$ in Lemma 3.1, we see that $N\left(a^{\prime}, b, c^{\prime}\right)$. For $t \in c / a$ we must have $\left(t+a^{\prime} b\right) c=t$, for otherwise the lattice generated by $a^{\prime}$, $b, c$, and $t$ has a sublattice isomorphic to $L_{10}$ (Fig. 13). Similarly, for $t^{\prime} \in\left(c+a^{\prime} b\right) /\left(a+a^{\prime} b\right)$ we must have $c t^{\prime}+a^{\prime} b=t^{\prime}$ to avoid $L_{8}$ (Fig. 14). Dually, for $t^{\prime} \in c^{\prime} / a^{\prime}$ and $t \in c^{\prime}(c+b) / a^{\prime}(c+b)$ we must have $t^{\prime}(c+b)+a^{\prime}=t^{\prime}$ and $\left(t+a^{\prime}\right)(c+b)=t$. Finally, for $t \epsilon$ $\left(c+a^{\prime} b\right) /\left(a+a^{\prime} b\right)$ and $t^{\prime} \in c^{\prime}(c+b) / a^{\prime}(c+b)$ we must have $\left(t+a^{\prime}(c+b)\right)\left(c+a^{\prime} b\right)=t$ and $t^{\prime}\left(c+a^{\prime} b\right)+a^{\prime}(c+b)=t^{\prime}$ in order to avoid $L_{6}$ (Fig. 15).

We conclude that the transpositions $t \rightarrow t+a^{\prime}$ and $t^{\prime} \rightarrow t c$ are isomorphisms between the quotients $c / a$ and $c^{\prime} / a^{\prime}$, and are inverses of each other, as was to be shown.

COROLLARY 3.3. If the $N$-quotient $c / a$ in $L$ projects onto a quotient $u / v$ then $u / v$ is an $N$-quotient, and the projectivity is an isomorphism. 
COROLLARY 3.4. If an $N$-quotient $c / a$ in $L$ projects weakly onto a quotient $u / v$, then a subquotient of $c / a$ projects onto $u / v$.

LEMMA 3.5. If $c_{i} / a_{i}, i=0,1,2$, are $N$-quotients in $L$ with $c_{0} / a_{0} \nearrow c_{1} / a_{1} \searrow c_{2} / a_{2}$ then $c_{0} / a_{0} \searrow c_{0} c_{2} / a_{0} a_{2} / c_{2} / a_{2}$.

Proof. We have $c_{1}=c_{0}+a_{1}=a_{1}+c_{2}$, hence by the semidistributivity of $L, c_{1}=a_{1}+c_{0} c_{2}$. It follows by Lemma 3.2 that

$$
a_{0}+c_{0} c_{2}=\left(a_{0}+c_{0} c_{2}+a_{1}\right) c_{0}=c_{1} c_{0}=c_{0}
$$

and, similarly, $\quad a_{2}+c_{0} c_{2}=c_{2}$. Also, $\quad a_{0}\left(c_{0} c_{2}\right)=c_{0} a_{1} c_{2}=a_{0} a_{2}$ and $a_{2}\left(c_{0} c_{2}\right)=a_{0} a_{2}$.

CoROLlaRY 3.6. If the $N$-quotient $c / a$ in $L$ projects onto a quotient $u / v$, then $c / a \nearrow x / y \searrow u / v$ for some quotient $x / y$.

Proof. Apply Corollary 3.3 and the dual of Lemma 3.5.

CoROLlaRY 3.7. If $c / a$ is an $N$-quotient in $L$, then con $(a, c)$ does not collapse any nontrivial quotient $u / v$ with $u \leqq a$ or $c \leqq v$.

4. Critial edges, Let $V$ be a variety that contains none of the lattices $M_{3}, L_{1}, L_{2}, \cdots, L_{12}$ and let $L \in V$ be a subdirectly irreducible, non-distributive lattice. Our aim in this section is to show that $L$ has a unique critical edge $c / a$ and that $c / a$ is also the only $N$-quotient of $L$. It follows that $L / \operatorname{con}(a, c)$ is distributive and that $L$ is locally finite.

LEMMA 4.1. If $c / a$ is a critical edge of $L$, then $c$ covers $a$, and $c / a$ is an $N$-quotient.

Proof. Since $L$ is non distributive and semidistributive, it has an $N$-quotient $u / v$. Since $\operatorname{con}(u, v)$ identifies $a$ and $c$, there exist elements $x_{0}, x_{1}, \cdots, x_{n} \in L$ with $c=x_{0}>x_{1}>\cdots>x_{n}=a$ such that $u / v$ projects weakly onto each of the quotients $x_{i} / x_{i+1}$. By Corollaries 3.3 and 3.4 , all the quotients $x_{i} / x_{i+1}$ are $N$-quotients, and, of course, they are all critical. Hence, all the congruence relations $\operatorname{con}\left(x_{i}, x_{i+1}\right)$ are equal, and by Corollary 3.7 this implies that $n=1$. Thus, $c / a$ is an $N$-quotient. To show that $c$ covers $a$ we again appeal to Corollary 3.7.

Lemma 4.2. All the $N$-quotients in $L$ are critical edges of $L$, and they are all projective to each other. 
Proof. Choose a critical edge $c / a$ of $L$. By the preceding lemma, $a \prec c$, and $c / a$ is an $N$-quotient. By Corollary 3.4, every $N$-quotient $u / v$ has a subquotient $u^{\prime} / v^{\prime}$ that is projective to $c / a$, and is therefore a critical edge of $L$. Furthermore, $u^{\prime} / v^{\prime}$ cannot be a proper subquotient of $u / v$, for if, say, $u^{\prime}<u$, then $\operatorname{con}\left(u, u^{\prime}\right)$ collapses $u^{\prime} / v^{\prime}$, contrary to Corollary 3.7. Thus $u / v=u^{\prime} / v^{\prime}$ is a critical edge of $L$ projective to $c / a$.

LEMMA 4.3. Let $\theta$ be the smallest non-trivial congruence relation on $L$. Then $L / \theta$ is distributive and, for all $u, v \in L$ with $u>v$, $\theta$ identifies $u$ and $v$ if and only if $u / v$ is an $N$-quotient.

Proof. By the preceding lemma, $\theta$ collapses all the $N$-quotients of $L$, whence it follows that $L / \theta$ cannot contain a pentagon. Since $L / \theta$ belongs to $\boldsymbol{V}$, it does not contain a diamond either, and it must therefore be distributive. The second part of the lemma follows from the fact that, by Lemmas 4.1 and 4.2 , the $N$-quotients in $L$ are precisely the critical edges.

The next step is to prove that $\operatorname{con}(a, c)$ idenitfies no two distinct elements other than $a$ and $c$.

Lemma 4.4. If c/a is a critical edge of $L$, then $a$ is meet irreducible and $c$ is join irreducible.

Proof. By Lemma 4.1, $a \prec c$ and $c / a$ is an $N$-quotient. Let us assume that $a$ is meet reducible; that is, $a=c d$ for some $d>a$. Then $\operatorname{con}(a, d)$ identifies $a$ and $c$, and, hence, there exist quotients $x_{i} / y_{i}, i=0,1, \cdots, n$, with $x_{0} / y_{0}=d / a, y_{n}=a$ and $x_{n} \geqq c$, such that, for $i<n, x_{i} / y_{i}$ transposes weakly up or down onto $x_{i+1} / y_{i+1}$. We assume that $n$ has been chosen as small as possible. Clearly, $n \geqq 2$.

The first two weak transpositions go one up and the other down, and the order cannot be reversed by replacing $x_{1} / y_{1}$ by another quotient. This is obvious when $n>2$, for if the order could be changed, then the sequence of quotients could be shortened by replacing $x_{1} / y_{1}$ and $x_{2} / y_{2}$ by a single quotient. Regarding the case $n=2$, we need only observe that we cannot have $d / a \searrow_{w} u / v \nearrow_{w} s / a$ with $s \geqq c$, for then $c \leqq u+a \leqq d$.

First, let us suppose that $d / a \nearrow_{w} x_{1} / y_{1} \searrow_{w} x_{2} / y_{2}$. By Lemma 2.2, there exist $a^{\prime}, b, c^{\prime} \in L$ with $N\left(a^{\prime}, b, c^{\prime}\right)$ such that either $b / b c^{\prime}$ is a subquotient of $d / a$, or elso $\left(a^{\prime}+b\right) / b$ transposes down onto a subquotient of $d / a$. In either case, $a \leqq b$. By Lemma $4.2 c / a$ and $c^{\prime} / a^{\prime}$ are projective, whence it follows by Lemma 3.2 that $N(a, b, c)$. However, this is impossible since $a \leqq b$.

Next, let $d / a \searrow_{w} x_{1} / y_{1} \nearrow_{w} x_{2} / y_{2}$. By the dual of Lemma 2.2 there 
exist $a^{\prime}, b, c^{\prime} \in L$ with $N\left(a^{\prime}, b, c^{\prime}\right)$ such that either $\left(a^{\prime}+b\right) / b$ is a subquotient of $d / a$, or else $b / b c^{\prime}$ transposes up onto a subquotient of $d / a$. As before, $N(a, b, c)$, thus $a \not b$, and $\left(a^{\prime}+b\right) / b$ cannot be a subquotient of $d / a$. Also, $b / b c^{\prime}$ cannot transpose up onto a subquotient of $d / a$, for this would imply that $a+b \leqq d$; hence, $c \leqq d$.

COROLlaRy 4.5. L has only one critical edge c/a, and con $(a, c)$ identifies no two distinct elements of $L$ other than $a$ and $c$.

Proof. By Lemmas 4.1 and 4.2, all the critical edges of $L$ are projective to each other, but by Lemma 4.4, a critical edge cannot be projective to any quotient distinct from itself. Hence, $L$ has only one critical edge. The second statement of the lemma follows by Lemma 4.3 .

CoRollary 4.6. L is locally finite.

Proof. If $\phi(n)$ is the order of a free distributive lattice with $n$ generators, then an $n$-generated sublattice of $L$ can have at most $\dot{\phi}(n)+1$ elements.

5. Proof of Theorem 1.1. Let $V$ be a variety that contains none of the lattices $M_{3}, L_{1}, L_{2}, \cdots, L_{15}$ and let $L \in V$ be a subdirectly irreducible, non-distributive lattice. Since any variety is determined by its finitely generated subdirectly irreducible members we may take $L$ to be finitely generated; whence, by Corollary $4.6, L$ is, in fact, finite. Let $c / a$ be the unique critical edge of $L$. To complete the proof of Theorem 1.1 it would suffice to show that $L$ must be a pentagon. This is the objective of this section.

LEMmA 5.1. There exists $b \in L$ such that $N(a, b, c), b c \prec a$ and $c \prec a+b$.

Proof. Choose $b \in L$ with $N(a, b, c)$ so that the quotient $(a+b) / b c$ is minimal. Given $c<t \leqq a+b$, we cannot have $b t=b c$, for then $t / c$ would be an $N$-quotient, contrary to the fact that $c / a$ is the only $N$-quotient in $L$. Letting $b^{\prime}=b t$, we therefore have $a<a+b^{\prime}$, and hence, $c \leqq a+b^{\prime}$ by the meet irreducibility of $a$. Thus $N\left(a, b^{\prime}, c\right)$, and in view of the choice of $b$ this yields $a+b^{\prime}=$ $a+b$; hence, $t=a+b$. Thus, $c \prec a+b$ and, by duality, $b c \prec b$.

LEMMA 5.2. The elements $a$ and $c$ are doubly irreducible.

Proof. By the preceding lemma we can choose $b \in L$ with 
$N(a, b, c), b c<a$ and $c \prec a+b$. According to Lemma 4.4, $a$ is meet irreducible and $c$ is join irreducible, so that by duality it suffices to show that $c$ is meet irreducible. If this is not the case, then there exists $d \in L$ with $c=(a+b) d$ and $c<d$. As in the proof of Lemma 4.4, we see that there exists a quotient $u / v$ such that one and only one of the following two statements holds:

(i) $d / c$ transpose weakly up onto a quotient that transposes weakly down onto $u / v$;

(ii) $d / c$ transposes weakly down onto a quotient that transposes weakly up onto $u / v$.

We shall show that either case leads to a contradiction.

Case (i). By Lemma 2.2 and the fact that $c / a$ is the only $N$-quotient in $L$, there exists $b^{\prime} \in L$ with $N\left(a, b^{\prime}, c\right)$ such that either $b^{\prime} / b^{\prime} c$ is a subquotient of $d / c$, or else $\left(a+b^{\prime}\right) / b^{\prime}$ transposes weakly down onto a subquotient of $d / c$. Regardless of which alternative applies, we have $c \leqq b^{\prime}$, contrary to the fact that $N\left(a, b^{\prime}, c\right)$.

Case (ii). Using the dual of Lemma 2.2, we obtain $b^{\prime} \in L$ with $N\left(a, b^{\prime}, c\right)$ such that either $\left(a+b^{\prime}\right) / b^{\prime}$ is a subquotient of $d / c$ or else $b^{\prime} / b^{\prime} c$ transposes up onto a subquotient of $d / c$. The former case is excluded by the fact that $c \leqq b^{\prime}$. In the latter case $b^{\prime} \leqq d$, and hence, $(a+b)\left(a+b^{\prime}\right)=c$. The elements $a, b$, and $b^{\prime}$ generate a sublattice $K$ of $L$ with the property that the congruence relation $\theta=\operatorname{con}(a, c)$ identifies no two distinct elements of $K$ except $a$ and $c$, and that $K / \theta$ is distributive. Since $\theta$ identifies the elements $(a+b)\left(a+b^{\prime}\right)=c$ and $a, K / \theta$ is a homomorphic image of the lattice in Fig. 16.

Let $a>a b^{\prime}+a b$. Then $\theta$ does not identify $c$ and $a b^{\prime}+a b$. Also, $\theta$ does not identify $c$ with either $a+b$ or $a+b^{\prime}$. Consequently, $a+b, a+b^{\prime}$, and $b+b^{\prime}$ generate, in this case, an eight element Boolean algebra. Then $a+b, a+b^{\prime}, b+b^{\prime}$, and $a$ generate a lattice isomorphic to $L_{13}$.

Thus, we must have $a=a b+a b^{\prime}$, and $K / \theta$ must be a homomorphic image of the lattice in Fig. 17. Actually, this homomorphism must be an isomorphism, since no two of the elements $b, b^{\prime}$, and $c$ are comparable modulo $\theta$. However, this implies that $K$ is isomorphic to $L_{15}$, so this too leads to a contradiction.

Lemma 5.3. $L$ is a pentagon.

Proof. By Lemma 5.1 we can choose $b \in L$ so that $N(a, b, c)$, $b c \prec a$ and $c<a+b$. Let $u=a+b$ and $v=b c$. 
We claim that $u(s+t)=u s+u t$ for all $s, t \in L$. By Lemma 4.3 and Corollary 4.5, this holds modulo $\operatorname{con}(a, c)$, and the only way the equation can fail is if $u(s+t)=c$ and $u s+u t=a$. Since $c$ is doubly irreducible $c=s+t$; hence, $s=c$ or $t=c$, so that $u s+u t=$ $c>a$.

Defining sot by $u s=u t$, we infer that $\phi$ is a congruence relation on $L$. Since $\phi$ does not identify $a$ and $c, \phi$ must be trivial. From this we infer that $t \leqq u$ for all $t \in L$, since $\phi$ always identifies $u+t$ with $u$. Similarly, $t \geqq v$ for all $t \in L$.

No element other than $a, c, u$, and $v$ is comparable with either $a$ or $c$, for if $t \leqq a$, then $t=a$ or $t=v$, while if $a<t$, then $c \leqq t$ by the meet irreducibility of $a$, and therefore, $t=c$ or $t=u$. If $t$ is not comparable with $a$ or $c$, then $a+t=u$ and $c t=v$, so that $N(a, t, c)$. From this, we infer that $v \prec b \prec u$, for if $b<t<u$, say, then $N(b, c, t)$, contrary to the fact that $c / a$ is the only $N$ quotient of $L$. Thus if $t \in L$ is distinct from $a, b, c, u$, and $v$, then $b+t=c+t=u$ and $b t=c t=v$, so that $b, c$, and $t$ generate a diamond.

6. Related results. While semidistributivity as applied to varieties of lattices, rather than individual lattices, is not equivalent to a conjunction of identities the next result shows that semidistributivity is equivalent to the disjunction of countably many identities.

THEOREM 6.1. Let $y_{0}=y, z_{0}=z$ and, for $n=0,1,2, \cdots$, let $y_{n+1}=y+x z_{n}, z_{n+1}=z+x y_{n}$. Then a variety $V$ is semidistributive if and only if, for some $n=0,1,2, \cdots, x(y+z)=x y_{n}=x z_{n}$ and its dual hold in $\boldsymbol{V}$.

Proof. If $L \in V$ is not semidistributive then there are elements $x, y, z$ in $L$ such that $x y=x z<x(y+z)$ say. Then, for all $n=$ $0,1,2, \cdots, y_{n}=y$ and $z_{n}=z$ whence $x y_{n}=x z_{n}<x(y+z)$.

Conversely, let us suppose that $V$ is a semidistributive variety. It suffices to show that, for some $n, x(y+z)=x y_{n}=x z_{n}$ in the free lattice $F_{V}(3)$ of $V$ generated by $x, y$, and $z$. In $F_{V}(3)^{\sigma}$ let $y_{\infty}$ be the join of the elements $y_{n}$ and let $z_{\infty}$ be the join of the elements $z_{n}$. Then $x y_{n} \leqq x z_{n+1}$ and $x z_{n} \leqq x y_{n+1}$ so that $x y_{\infty}=x z_{\infty}$. Now, $y_{n}+z_{n}=$ $y+z$, and semidistributivity implies that $x(y+z)=x y_{\infty}=x z_{\infty}$. It follows that, for some $n, x(y+z)=x y_{n}=x z_{n}$.

The proof of Theorem 6.1 yields the next result.

COROLlary 6.2. A variety $\boldsymbol{V}$ of lattice is semidistributive if 
and only if the lattices $F_{V}(3)^{\sigma}$ and $F_{V}(3)^{\pi}$ are semidistributive.

As we mentioned at the outset the problem of finding a set of identities which describes a given variety is usually quite difficult. This task was accomplished by R. McKenzie [8] in the case of the smallest non-modular variety $N$. Once these identities are exhibited, however, the matter of verifying that they describe precisely $N$ is, in view of Theorem 1.1, a simple computation.

THEOREM 6.3. $\quad \boldsymbol{N}$ is precisely the class of all lattices satisfying the two identities.

$$
x(y+z)(y+w) \leqq x(y+z w)+x z+x w
$$

and

$$
x(y+z(x+w))=x(y+x z)+x(x y+z w) .
$$

A lattice $L$ is said to satisfy $(W)$ if, for all $x, y, u, v \in L$, $x y \leqq u+v$ implies that either $x y \leqq u$ or $x y \leqq v$ or $x \leqq u+v$ or $y \leqq u+v$. It is easy to verify that each of the lattices $M_{3}, L_{1}, L_{2}, \cdots, L_{15}$ satisfies $(W)$. According to a result of B.A. Davey and B. Sands [3], every finite lattice satisfying $(W)$ is a retract of any finite lattice of which it is a homomorphic image. On the other hand, each subdirectly irreducible member of a variety $\boldsymbol{L}$ generated by a finite lattice $L$ is a homomorphic image of a sublattice of $L[5]$. Combining these observations with Theorem 1.1 yields our final result.

THEOREM 6.4. Let $L$ be a finite non-modular lattice. If $L$ is not a member of the smallest non-modular variety then $L$ contains a sublattice isomorphic to one of $M_{3}, L_{1}, L_{2}, \cdots, L_{15}$.

\section{REFERENCES}

1. K. A. Baker, Equational classes of modular lattices, Pacific J. Math., 28 (1969), 9-15.

2. B. A. Davey, W. Poguntke and I. Rival, A characterization of semi-distributivity, Alg. Univ., 5 (1975), 72-75.

3. B. A. Davey and B. Sands, An application of Whiteman's condition to lattices with no infinite chains, Alg. Univ., 7 (1977), 171-178.

4. G. Grätzer, Equational classes of lattices, Duke Math. J., 33 (1966), 613-622.

5. B. Jónsson, Algebras whose congruence lattices are distributive, Math. Scand., 21 (1967), 110-121.

6. —_ Equational classes of lattices, Math. Scand., 22 (1968), 187-196.

7. R. McKenzie, Equational bases for lattice theories, Math. Scand., 27 (1970), 24-38.

8. - Equational bases and nonmodular lattice varieties, Trans. Amer. Math. Soc., 174 (1972), 1-43. 
9. I. Rival, Varieties of nonmodular lattices, Notices Amer. Math. Soc., 23 (1976), A-420.

Received November 9, 1976 and in revised form March 21, 1978. The work of the first author was supported by NSF Grant MCS 76-06447; the work of the second author was supported by National Research Council Operating Grant A4077.

VANDERBILT UNIVERSITY

NASHVILLE, TN 37235

AND

The University of Calgary

Calgary, Alberta 


\section{PACIFIC JOURNAL OF MATHEMATICS}

\section{EDITORS}

Donald BABBITT (Managing Editor)

University of California

Los Angeles, CA 90024

HUGo RossI

University of Utah

Salt Lake City, UT 84112

C. C. MOORE and ANDREW OGG

University of California

Berkeley, CA 94720

\section{J. DuGUNDJI}

Department of Mathematics

University of Southern California

Los Angeles, CA 90007

R. FINN and J. Milgram

Stanford University

Stanford, CA 94305

ASSOCIATE EDITORS
E. F. BECKENBACH
B. H. NeumanN
F. WOLF
K. YoSHIDA

\section{SUPPORTING INSTITUTIONS}

\author{
UNIVERSITY OF BRITISH COLUMBIA \\ CALIFORNIA INSTITUTE OF TECHNOLOGY \\ UNIVERSITY OF CALIFORNIA \\ MONTANA STATE UNIVERSITY \\ UNIVERSITY OF NEVADA, RENO \\ NEW MEXICO STATE UNIVERSITY \\ OREGON STATE UNIVERSITY \\ UNIVERSITY OF OREGON
}

\author{
UNIVERSITY OF SOUTHERN CALIFORNIA \\ STANFORD UNIVERSITY \\ UNIVERSITY OF HAWAII \\ UNIVERSITY OF TOKYO \\ UNIVERSITY OF UTAH \\ WASHINGTON STATE UNIVERSITY \\ UNIVERSITY OF WASHINGTON
}

The Supporting Institutions listed above contribute to the cost of publication of this Journal, but they are not owners or publishers and have no responsibility for its content or policies.

Mathematical papers intended for publication in the Pacific Journal of Mathematics should be in typed form or offset-reproduced, (not dittoed), double spaced with large margins. Please do not use built up fractions in the text of the manuscript. However, you may use them in the displayed equations. Underline Greek letters in red, German in green, and script in blue. The first paragraph or two must be capable of being used separately as a synopsis of the entire paper. Please propose a heading for the odd numbered pages of less than 35 characters. Manuscripts, in triplicate, may be sent to any one of the editors. Please classify according to the scheme of Math. Reviews, Index to Vol. 39. Supply name and address of author to whom proofs should be sent. All other communications should be addressed to the managing editor, or Elaine Barth, University of California, Los Angeles, California, 90024.

50 reprints to each author are provided free for each article, only if page charges have been substantially paid. Additional copies may be obtained at cost in multiples of 50 .

The Pacific Journal of Mathematics is issued monthly as of January 1966. Regular subscription rate: $\$ 84.00$ a year (6 Vols., 12 issues). Special rate: $\$ 42.00$ a year to individual members of supporting institutions.

Subscriptions, orders for numbers issued in the last three calendar years, and changes of address should be sent to Pacific Journal of Mathematics, P.O. Box 969, Carmel Valley, CA 93924, U.S.A. Older back numbers obtainable from Kraus Periodicals Co., Route 100, Millwood, NY 10546.

PUBLISHED BY PACIFIC JOURNAL OF MATHEMATICS, A NON-PROFIT CORPORATION

Printed at Kokusai Bunken Insatsusha (International Academic Printing Co., Ltd.). 8-8, 3-chome, Takadanobaba, Shinjuku-ku, Tokyo 160, Japan.

Copyright (C) 1979 by Pacific Journal of Mathematics Manufactured and first issued in Japan 


\section{Pacific Journal of Mathematics}

\section{Vol. 82 , No. 2 \\ February, 1979}

Krishnaswami Alladi and Paul Erdős, On the asymptotic behavior of large prime

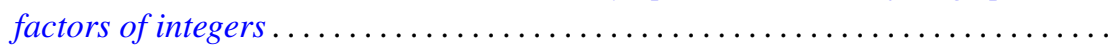

Alfred David Andrew, A remark on generalized Haar systems in $L_{p}$,

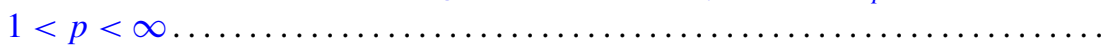

John M. Baker, A note on compact operators which attain their norm . . ........

Jonathan Borwein, Weak local supportability and applications to

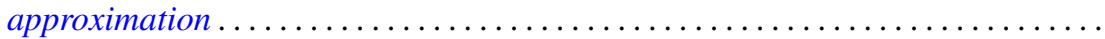

Tae Ho Choe and Young Soo Park, Wallman's type order compactification ........

Susanne Dierolf and Ulrich Schwanengel, Examples of locally compact

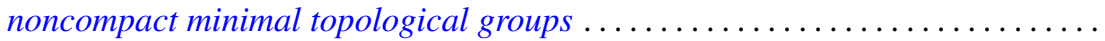

Michael Freedman, A converse to (Milnor-Kervaire theorem) $\times R$ etc. . . . . . . .

George Golightly, Graph-dense linear transformations ..................

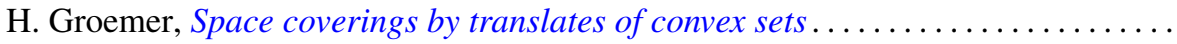

Rolf Wim Henrichs, Weak Frobenius reciprocity and compactness conditions in

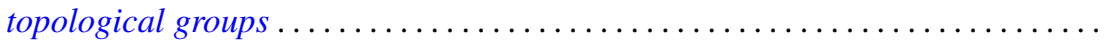

Horst Herrlich and George Edison Strecker, Semi-universal maps and universal

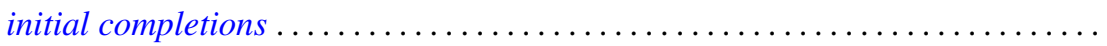

Sigmund Nyrop Hudson, On the topology and geometry of arcwise connected,

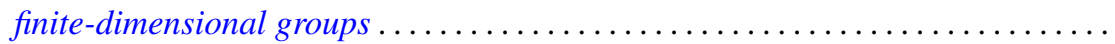

K. John and Václav E. Zizler, On extension of rotund norms. II .............

Russell Allan Johnson, Existence of a strong lifting commuting group of transformations. II.

Bjarni Jónsson and Ivan Rival, Lattice varieties covering the smallest nonmodular variety

Grigori Abramovich Kolesnik, On the order of Dirichlet L-functions .

Robert Allen Liebler and Jay Edward Yellen, In search of nonsolvable groups of

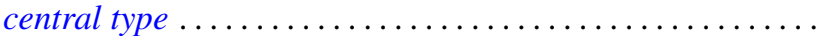

Wilfrido Martínez T. and Adalberto Garcia-Maynez Cervantes, Unicoherent plane Peano sets are $\sigma$-unicoherent ...

M. A. McKiernan, General Pexider equations. I. Existence of injective

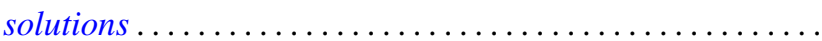

M. A. McKiernan, General Pexider equations. II. An application of the theory of webs.

Jan K. Pachl, Measures as functionals on uniformly continuous functions . .

Lee Albert Rubel, Convolution cut-down in some radical convolution algebras ...

Peter John Slater and William Yslas Vélez, Permutations of the positive integers

with restrictions on the sequence of differences. II . . .

Raymond Earl Smithson, A common fixed point theorem for nested spaces ....

Indulata Sukla, Generalization of a theorem of McFadden .... . . .

Jun-ichi Tanaka, A certain class of total variation measures of analytic measures.

Kalathoor Varadarajan, Modules with supplements .............. 